“C 2010 IEEE. Personal use of this material is permitted. Permission from IEEE must be obtained for all other uses, in any current or future media, including reprinting/republishing this material for advertising or promotional purposes, creating new collective works, for resale or redistribution to servers or lists, or reuse of any copyrighted component of this work in other works.” 


\section{Fault Diagnosis of Transformer using Association Rule Mining and Knowledge Base}

\author{
Tiefeng Zhang \\ School of Electric\&Electronic Engineering \\ North China Electric Power University \\ Baoding, China \\ ncepuztf@126.com
}

Jie Lu

DeSI lab, QCIS center

Faculty of Engineering and Information Technology, University of Technology Sydney

PO BOX 123, Broadway, NSW 2007, Australia

jielu@it.uts.edu.au

\author{
Guangquan Zhang \\ DeSI lab, QCIS center \\ Faculty of Engineering and Information Technology, \\ University of Technology Sydney \\ PO BOX 123, Broadway, NSW 2007, Australia \\ zhangg@it.uts.edu.au
}

\author{
Qian Ding \\ School of Electric\&Electronic Engineering \\ North China Electric Power University \\ Baoding, China \\ ncepudingqian@126.com
}

\begin{abstract}
Association rule mining makes interesting associations and/or correlations among large sets of data. Those associations can be refined as decision rules to be used and stored in a knowledge base system. In this paper, an approach based on association rule and knowledge base is proposed and implemented in the fault diagnosis of a transformer system. According to the features of association rule, the Apriori algorithm is adopted and modified to generate decision rules from power transformer information for building knowledge base, then the rules can be refined to diagnose the fault of the transformer through reasoning, and a prototype system is developed. This approach based on association rule is described in detail and the application is illustrated by an example. A comparison with the IEC (International Electrotechnical Commission) three-ratio method shows the proposed method can provide better accuracy in performance.
\end{abstract}

Keywords-Apriori; association rule mining; knowledge base; dissolved gas analysis; fault diagnosis; power transformer

\section{INTRODUCTION}

The power transformer is a major apparatus in a power system. Transformer faults have caused huge economic losses and brought about adverse social impacts in the past. Early diagnosis to detect the incipient faults is necessary to avoiding catastrophic failures and costly outages.

To a large extent, the gas component and content dissolved in the transformer oil indicate the insulation aging and fault degree of the transformer, so the diagnosis of oilimmersed transformers using the dissolved gas analysis (DGA) technique has been well accepted and widely used, and various methods applied for the DGA have been developed to interpret DGA results such as IEC ratio code[1] and the Rogers method [2], their limitation is that the predefined criteria are established upon empirical studies. In some cases, measured gas concentrations or ratios may be incomplete and thus do not fit within the predefined criteria. As a result, faults that occur inside transformers may not be identifiable. Other methods include rough sets theory [3,4], fuzzy theory [5,6], support vector machine [7], artificial neural network [8]-[11], Bayesian network [12], grey relational analysis [13], extension theory [14], fuzzy entropy [15], Case-based Reasoning Theory [16], decision tree [17], Kernel-Based Possibilistic Clustering [18], Expert System [19] and hybrid methods [20]-[24], all of these methods have been developed to improve diagnosis accuracies. However, these methods sometimes fail to determine the fault type, and the workers onsite often use different methods or other related information about the transformer to do such judge fault. Therefore, exploring new ways to resolve this early warning problem is still a challenge.

Association rule mining (ARM) is one of the data mining techniques used to extract hidden knowledge from data sets, which can be used by an organization's decision makers to improve overall profit [25]. An ARM-based DGA approach [26] has been presented to fault diagnosis of power transformers, the result shows the approach is capable of generating a number of meaningful association rules and can achieve higher fault diagnosis accuracy, but owing to small number of samples used, more experiments are expected to examine it.

ARM-based methods are applied in many fields. For customer relationship management (CRM), the technical analysis of customer using association rules can show the buying behavior, so that the managers of supermarket can adjust the layout of products to improve their services and profits. In the financial field, association rules technology is used in banking, securities, insurance, etc. and has discovered some important rules to support business decisions. In addition, the association rules technique also applies to the life sciences, information technology, industrial control, network security and so on.

For an oil-immersed power transformer, during the past forty years, dissolved gas analysis (DGA) has become universally accepted as the premier diagnostic tool for location of incipient faults in transformers with an 
assumption based on the idea that the gases produced during normal operation of oil filled circuit breakers (OCB) or load tap changers (LTC) would be more significant than gases produced by a fault process such as contact overheating. Therefore, there should be some association between the fault (the working state) and the dissolved gases.

In this study, we introduce the diagnosis accuracy of ARM-based DGA approach [26], and also explain how to incorporate knowledge base with ARM-based DGA to build a fault diagnosis system. This ARM-based approach consists of a rule discovery method modified by the Apriori algorithm [27] and a rule-based knowledge system [28, 29] for diagnosis of the power transformer fault. A comparison with the IEC three-ratio method is implemented to validate the effectiveness of the proposed approach. A knowledge base is established for incorporating more useful rules.

This paper is organized as follows. Section II introduces the concepts and notions of information tables, association rule and knowledge base. Based on these basic concepts and notions, Section III presents an association rule and knowledge-base approach, including how to pre-process the data, how to modify the Apriori algorithm to generate rules and refine them, and how to use rules to diagnose the fault of a transformer. In Section IV, a case based example illustrates the effectiveness of the proposed association rule approach in comparison with IEC three-ratio method. Conclusions and future work are provided in Section V.

\section{BASIC NOTIONS}

\section{A. Information tables and decision tables}

In general, an information table is a system to express knowledge which can be used to represent and process knowledge in machine learning, data mining and other related fields.

The definitions of information table and decision table have been described in [27].

A decision table is a special case of an information table.

A decision table can be seen as a special and important knowledge expression system. It shows that, when some conditions are satisfied, decisions, actions, operations, or controls can be made. Decision attributes in a decision table may, or may not, be unique. In the latter case, the decision table can be converted to one with unique decision attributes. Therefore, in this paper, we assume that there is only one decision attribute.

In this paper, an information table has the form of a decision table with condition attributes $C$, including the key gases (e.g. hydrogen $\left(\mathrm{H}_{2}\right)$, methane $\left(\mathrm{CH}_{4}\right)$, ethene $\left(\mathrm{C}_{2} \mathrm{H}_{6}\right)$, ethylene $\left(\mathrm{C}_{2} \mathrm{H}_{4}\right)$, acetylene $\left.\left(\mathrm{C}_{2} \mathrm{H}_{2}\right)\right)$ and decision attributes $D$ called "the working state". This table contains the fault records as raw data for extracting rules for diagnosis.

\section{B. Association rule}

Let $D$, the task-relevant data be a set of database transactions where each transaction $T$ is a set of items where each transaction is associated with an identifier, called TID. Let $A$ be a set of itemsets.

An association rule is an implication of the form $A \Rightarrow B$, where $A \subseteq I, B \subseteq I$, and $A \cap B=\varnothing$. The rule $A \Rightarrow B$ holds in the transaction set $D$ with support $s$, where $s$ is the percentage of transactions in $D$ that contain $A \cup B$. This is taken to be the probability, $P(A \cup B)$. The rule $A \Rightarrow B$ has confidence $c$ in the transaction set $D$ if $c$ is the percentage of transactions in $D$ containing $A$ that also contain $B$. This is taken to be the conditional probability, $P(B \mid A)$. That is, support $(A=>B)=P(A \cup B)$, confidence $(A=>B)=\mathrm{P}(B \mid A)$.

Rules that satisfy both a minimum support threshold (min_sup) and a minimum confidence threshold (min_conf) are called strong rules. If an itemset satisfies minimum support, then it is a frequent itemset.

Apriori [27] is a classical algorithm to find association rules of item-set, which mines frequent itemsets by using a circulatory method to search frequent itemsets that produces $(k+1)$-item-sets from $k$-item-sets. After scanning the database, the algorithm begins to circulate. Each time, it connects two $(k-1)$ itemsets, and produces frequent $k$ itemsets after the riddling of Apriori property, that is "each subsets of the frequent itemsets also should be frequent" as well as the minimum support degree. The Apriori ends till the frequent itemsets can't be produced.

The procedure using Apriori algorithm can be described as follows:

For each frequent item-sets $L$, produces all subsets.

For each non-null subset $S$ of $L$, generates an association rule " $s \rightarrow(l-s)$ ", if it's confidence degree is not less than min_conf.

This is the classic algorithm for association rules. In this paper, we modify this method in order to build the knowledge base of an expert system.

\section{Knowledge base}

Knowledge base is a product of artificial intelligence and database technology, also a collection of knowledge and data. The keys to the development of technology are the knowledge acquisition, knowledge storage and representation, knowledge reasoning.

1) Knowledge acquisition: Knowledge acquisition is a process whereby the knowledge from external knowledge sources is converted to the knowledge-based system. The object of knowledge acquisition, including the knowledge of domain experts and other relevant knowledge, must go through a refining process to improve problem-solving abilities.

The means of knowledge acquisition usually contains two methods, which are interactive access and natural language acquisition. In this paper, the rules will be obtained by association rule, and then processed to become knowledge by a domain expert.

The rules above are based on support and confidence which are objective assessment of conditions. These rules must be processed by subjective evaluation with domain knowledge, to then become useful knowledge.

After knowledge acquisition, the rules can be stored in the knowledge base, and then used to diagnose by reasoning.

2) Knowledge storage and representation: To place the 
knowledge into the knowledge Base, it needs a variable table and the rule table established.

- Variable table: In this study, we choose $\mathrm{H}_{2}, \mathrm{CH}_{4}$, $\mathrm{C}_{2} \mathrm{H}_{6}, \mathrm{C}_{2} \mathrm{H}_{4}, \mathrm{C}_{2} \mathrm{H}_{2}$ as the variables of the knowledge base, more variables can be added.

- Rule table: Through establishing rule table, we can carry reasoning process by matching the rules with the facts (the variable bound values).

The representation of knowledge contains the first order predicates logic, production rule for semantic network, production rule representation, and frame theory representation and so on. In this study, production rule representation is the best choice to express the knowledge.

The form of this method is: $P \rightarrow Q$.

Variable is the basic unit of the rules. In a relational database, each variable is a record in the table, and the attributes are the fields in the database. The fields are as shown in Table I.

TABLE I. FIELDS OF VARIABLE TABLE

\begin{tabular}{|l|l|l|}
\hline \multicolumn{1}{|c|}{ Fields } & Type & \multicolumn{1}{c|}{ Explain } \\
\hline VariableID & Integer & Number of variables \\
\hline Chinesename & Text & Chinese name of variables \\
\hline Englishname & Text & $\begin{array}{l}\text { English name, Represent the variables in the } \\
\text { expression values }\end{array}$ \\
\hline Max & Float & The max of variable values \\
\hline Min & Float & The min of variable values \\
\hline
\end{tabular}

$\mathrm{H}_{2}, \mathrm{CH}_{4}, \mathrm{C}_{2} \mathrm{H}_{4}, \mathrm{C}_{2} \mathrm{H}_{2}, \mathrm{C}_{2} \mathrm{H}_{6}$ are the variables with No. 0, 1, 2, 3 and 4. Max and Min denote the maximal value and minimal value, the gas data between Max and Min is valid.

Rule table is the basis for problem solving, and their knowledge of the integrity, consistency, accuracy, flexibility, and the rationality of knowledge organization, has an important impact on the operating efficiency of the system. The fields of the rule table are shown in Table II.

3)Knowledge reasoning: In this study, we use forward reasoning for inference. After the user inputs the gas values of a new power transformer, the reasoning process is as follows.

First, bind corresponding variables with these values by searching the variable table (Table I) with the known facts.

Secondly, match antecedents of each rule (Table II) against known facts to produce a set of applicable rules.

Finally, choose one applicable rules to apply (e.g. first rule which produces new facts), adding new facts to the fact-base.

TABLE II. FIELDS OF RULE TABLE

\begin{tabular}{|l|l|l|}
\hline \multicolumn{1}{|c|}{ Fields } & \multicolumn{1}{c|}{ Type } & \multicolumn{1}{c|}{ Explain } \\
\hline Ruleid & Integer & Number of variables \\
\hline Cls_variableid & Text & The condition variables \\
\hline Clause_vars & Text & $\begin{array}{l}\text { The antecedent variables separated by } \\
\text { comma }\end{array}$ \\
\hline Opts & Text & $\begin{array}{l}\text { Determine symbols( }>=,>,<,<=, \quad== \\
!=)\end{array}$ \\
\hline Decidevalues & Text & Determine value, separated by comma \\
\hline Expression & Text & Explanation of rules \\
\hline
\end{tabular}

\section{THE PROPOSED APPROACH BASED ON ASSOCIATION RULE AND KNOWLEDGE BASE}

In this section, a proposed approach based on association rule and knowledge base is described, and the procedure of the approach is given in detail.

To use association rules for transformer fault diagnosis, we need to choose the most representative attributes of variables and collect data records, then pre-process them to be suitable for using ARM. Refined rules generated by ARM will be stored in the knowledge base, and when a new power transformer's DGA record is provided, the rules in knowledge will be used to diagnose the working state of the power transformer by reasoning. The workflow chart of the approach is shown in Fig.1.

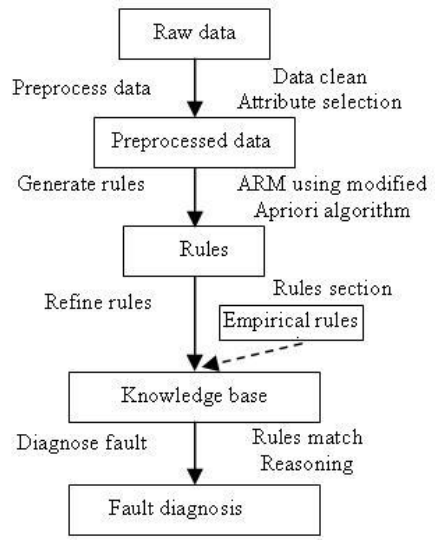

Figure 1 The workflow chart

Some steps are detailed as follows.

\section{A. Pre-process data}

In the real world, incomplete, noisy, and inconsistent data are common in the data we wish to analyze, so the first thing we should do is data pre-processing.

Data pre-processing includes data cleaning, data integration, data transformation, and data reduction [30].

Data reduction obtains a reduced representation of the data set that is much smaller in volume, yet produces the same (or almost the same) analytical results. There are a number of strategies for data reduction, such as data cube aggregation, attribute subset selection, dimensionality reduction, numerosity reduction, and discretization can be used to obtain a reduced representation of the data while minimizing the loss of information content. Data discretization is a form of data reduction that is very useful for the automatic generation of concept hierarchies from numerical data.

Data discretization techniques can be used to reduce the number of values for a given continuous attribute by dividing the range of the attribute into intervals. Interval labels can then be used to replace actual data values. Replacing numerous values of a continuous attribute by a small number of interval labels thereby reduces and simplifies the original data. This leads to a concise, easy-touse, knowledge-level representation of mining results.

In this study, we will use a rough set and boolean 
reasoning approach [31] to solve this of real value attributes quantity problem, the approach has been verified for its ability of improving classification and accepted widely in practice.

The values of each gas are divided into several intervals and different gases have different number of intervals.

\section{B. Generate Rules by Modified Apriori algorithm}

In the Apriori algorithm, two frequent $(k-1)$-item are usually linked if they have the same front $(k-2)$ items. For example, from link "1.3, 2.1, 3.1, 4.2, 5.2, 6.1" and "1.3, 2.1, 3.1, 4.2, 5.1, 6.1", we can get " $1.3,2.1,3.1,4.2,5.1$, $5.2,6.1$ ". However, this is not, in fact, reasonable, because "5.1" and "5.2" are two possible values of the same attribute,so they can't exist simultaneously. In the proposed approach, we modify the link rules, if two frequent $(k-1)$ items have the same front $(k-2)$ items and their $(k-1)$ th items aren't possible values of the same attribute, we link them. After the linking work and the set of the min_sup, we get the frequent k-itemsets which satisfies both min_conf and min_sup. From the above example, we get the frequent 5itemsets finally, as shown in Table III.

TABLE III. EXAMPLE: THE FREQUENT 5-ITEMSETS

\begin{tabular}{|c|c|}
\hline Item & Support \\
\hline $1.5,2.3,4.3,5.4,6.1$ & 4 \\
\hline $1.5,3.3,4.3,5.4,6.1$ & 5 \\
\hline $1.6,2.2,3.2,4.3,6.2$ & 6 \\
\hline $1.6,2.2,4.3,5.5,6.2$ & 4 \\
\hline $1.6,2.3,3.3,4.3,6.1$ & 5 \\
\hline $1.6,2.3,3.4,4.3,6.1$ & 6 \\
\hline $1.6,2.3,4.3,5.2,6.1$ & 5 \\
\hline $1.6,3.2,4.3,5.4,6.2$ & 4 \\
\hline $2.1,3.1,4.1,5.5,6.2$ & 4 \\
\hline $2.2,3.2,4.3,5.5,6.2$ & 4 \\
\hline $2.3,3.3,4.3,5.4,6.1$ & 6 \\
\hline $2.3,3.4,4.3,5.4,6.1$ & 4 \\
\hline
\end{tabular}

In the classic association algorithm, for all non-null subsets of frequent itemsets $L$ and each nominal subset $S$, we produce an association rule " $s \rightarrow(l-s)$ " if its confidence degree is not less than min_conf.

For the knowledge base, the expected result by ARM is that these rules with some condition attributes are contained in antecedents with a decision attribute in consequents, but not all of the association rules like this. We modified this part of Apriori to obtain these expected rules. According to the important feature that the non-frequent itemsets' subset is also non-frequent, we only need to calculate the confidence degree of the subset except for the consequent of decision rule. For example, to the frequent itemsets " 1.5 , $2.3,4.3,5.4,6.1$ ", we only need to calculate the confidence degree of " $1.5,2.3,4.3,5.4$ ", that is, $\mathrm{s}=$ " $1.5,2.3,4.3,5.4$ " (Because 6.1 is the consequent of decision rule). So, when it satisfies the rule that its confidence is more than min_conf. we can produce the rule $s \rightarrow(l-s)$, i.e. " $1.5,2.3,4.3$, $5.4 \rightarrow 6.1$ ".

\section{Refine Rules and add rules for knowledge base}

In Section B, through the ARM process, we find a lot of rules, but not every rule is appropriate, so we need to check them to keep the desired rules. In this study, this post process work is undertaken by a domain expert or knowledge worker.

The rules generated by ARM will not be enough for diagnosis, and some empirical rules from on-site inspection are also useful, so using a knowledge base could be more extendable and feasible in practice, as we can input those empirical rules into the knowledge base.

\section{Diagnose fault using rules}

The reasoning is mainly achieved by rule matching. Once a DGA record data or related data of a power transformer is provided, these values will be bound with corresponding variables and enter the reasoning process described in the knowledge reasoning section. After that, the fault type of the provided power transformer will be determined.

\section{A CASE STUDY}

A case study using the proposed approach is presented in this paper, including fault and normal data of power transformers, 127 training data sets is selected randomly for generating rules, and other 73 test data sets are used to validate the proposed approach using the obtained rules.

As we mentioned above, DGA records are chosen in this study, including the five dissolved gases: $\mathrm{H}_{2}, \mathrm{CH}_{4}, \mathrm{C}_{2} \mathrm{H}_{6}$, $\mathrm{C}_{2} \mathrm{H}_{4}, \mathrm{C}_{2} \mathrm{H}_{2}$. Table IV shows an excerpt of raw data.

TABLE IV. AN EXCERPT OF RAW DATA

\begin{tabular}{|c|c|c|c|c|c|}
\hline \multicolumn{5}{|c|}{ Gas } & \multirow{2}{*}{ Fault type } \\
\hline $\boldsymbol{H}_{\mathbf{2}}$ & $\boldsymbol{C H}_{\boldsymbol{4}}$ & $\boldsymbol{C}_{\mathbf{2}} \boldsymbol{H}_{\mathbf{6}}$ & $\boldsymbol{C}_{\mathbf{2}} \boldsymbol{H}_{\boldsymbol{4}}$ & $\boldsymbol{C}_{\boldsymbol{2}} \boldsymbol{H}_{\mathbf{2}}$ & \\
\hline 143 & 305 & 721 & 78 & 2 & Hyperthermia and superheating \\
\hline 173 & 334 & 172 & 813 & 37.7 & Hyperthermia and superheating \\
\hline 127 & 107 & 11 & 154 & 224 & High energy discharging \\
\hline 200 & 48 & 14 & 117 & 131 & High energy discharging \\
\hline 181 & 262 & 41 & 28 & 0 & Medium temperature and superheating \\
\hline 98 & 155 & 54 & 300 & 1.5 & Medium temperature and superheating \\
\hline 2781 & 1293 & 248 & 23 & 84 & Low energy discharging \\
\hline 200 & 50 & 40 & 50 & 6 & Low energy discharging \\
\hline 63.1 & 16.7 & 8.3 & 9.3 & 6.1 & Normal \\
\hline 87 & 41 & 10.1 & 72 & 1 & Normal \\
\hline
\end{tabular}

Discretization is the main task in processing data. Using the rough set and boolean reasoning approach (described detailly in [31]), we implement discretization function based on a toolkit named ROSETTA [32] in our prototype system. By this system, $\mathrm{H}_{2}$ can be divided into 8 intervals, denoted as 1.1-1.8 and $\mathrm{CH}_{4} 、 \mathrm{C}_{2} \mathrm{H}_{6} 、 \mathrm{C}_{2} \mathrm{H}_{4} 、 \mathrm{C}_{2} \mathrm{H}_{2}$ can be divided into several intervals. Different gases have different number of intervals. The discretization results are shown in Table V.

In this study, the working states of transformers include hyperthermia and superheating, high energy discharging, medium temperature and superheating, low energy discharging and normal. 
TABLE V. THE DISCRETIZATION RESULTS

\begin{tabular}{|c|c|c|c|c|}
\hline H2 & CH4 & C2H6 & C2H4 & C2H2 \\
\hline$[0,20)$ & {$[0,79)$} & {$[0,7)$} & {$[0,26.1)$} & {$[0,1.1)$} \\
\hline$[20,25)$ & {$[79,256)$} & {$[7,15)$} & {$[26.1,76.9)$} & {$[1.1,3.6)$} \\
\hline$[25,40)$ & {$[256, \infty)$} & {$[15,116)$} & {$[76.9, \infty)$} & {$[3.6,6.1)$} \\
\hline$[40,51)$ & & {$[116, \infty)$} & & {$[6.1,20.9)$} \\
\hline$[51,89)$ & & & & {$[20.9, \infty)$} \\
\hline$[89,191)$ & & & & \\
\hline$[191,318)$ & & & & \\
\hline$[318, \infty)$ & & & & \\
\hline
\end{tabular}

We used the modified Apriori algorithm to generate rules. For each "working state", we adjust the support and the confidence till we can get at least one rule or more when the support or confidence changes in the downward direction. During this process, a domain expert evaluates the generated rules and decides the rules to be kept by their domain knowledge and empirical evidence. In this study, we set min_sup $=0.03$ and min_conf $=0.6$, the refined rules are shown in Table VI.

TABLE VI. REFINED RULES

\begin{tabular}{|c|c|c|}
\hline Rules & Sup. & Conf. \\
\hline $\begin{array}{c}51<\mathrm{H} 2<89 \wedge \mathrm{CH} 4>256 \wedge \mathrm{C} 2 \mathrm{H} 4>76.9 \wedge \\
6.1<\mathrm{C} 2 \mathrm{H} 2<20.9-->\text { hyperthermia and superheating }\end{array}$ & $3.15 \mathrm{E}-02$ & 1 \\
\hline $\begin{array}{c}51<\mathrm{H} 2<89 \wedge 15<\mathrm{C} 2 \mathrm{H} 6<116 \wedge \mathrm{C} 2 \mathrm{H} 4>76.9 \wedge \\
6.1<\mathrm{C} 2 \mathrm{H} 2<20.9-->\text { hyperthermia and superheating }\end{array}$ & 3.94E-02 & 1 \\
\hline $\begin{array}{c}89<\mathrm{H} 2<191 \wedge 79<\mathrm{CH} 4<256 \wedge 7<\mathrm{C} 2 \mathrm{H} 6<15 \wedge \\
\mathrm{C} 2 \mathrm{H} 4>76.9-->\text { high energy discharging }\end{array}$ & 4.72E-02 & 1 \\
\hline $\begin{array}{c}89<\mathrm{H} 2<191 \wedge 79<\mathrm{CH} 4<256 \wedge \mathrm{C} 2 \mathrm{H} 4>76.9 \wedge \\
\mathrm{C} 2 \mathrm{H} 2>20.9-->\text { high energy discharging }\end{array}$ & $3.15 \mathrm{E}-02$ & 1 \\
\hline $\begin{array}{l}89<\mathrm{H} 2<191 \wedge \mathrm{CH} 4>256 \wedge 15<\mathrm{C} 2 \mathrm{H} 6<116 \wedge \\
\mathrm{C} 2 \mathrm{H} 4>76.9-->\text { hyperthermia and superheating }\end{array}$ & $3.94 \mathrm{E}-02$ & 1 \\
\hline $\begin{array}{c}89<\mathrm{H} 2<191 \wedge \mathrm{CH} 4>256 \wedge \mathrm{C} 2 \mathrm{H} 6>116 \wedge \\
\mathrm{C} 2 \mathrm{H} 4>76.9-->\text { hyperthermia and superheating }\end{array}$ & $6.30 \mathrm{E}-02$ & 0.75 \\
\hline $\begin{array}{c}89<\mathrm{H} 2<191 \wedge \mathrm{CH} 4>256 \wedge \mathrm{C} 2 \mathrm{H} 4>76.9 \wedge \\
1.1<\mathrm{C} 2 \mathrm{H} 2<3.6-->\text { hyperthermia and superheating }\end{array}$ & $3.94 \mathrm{E}-02$ & 1 \\
\hline $\begin{array}{c}89<\mathrm{H} 2<191 \wedge 7<\mathrm{C} 2 \mathrm{H} 6<15 \wedge \mathrm{C} 2 \mathrm{H} 4>76.9 \wedge \\
6.1<\mathrm{C} 2 \mathrm{H} 2<20.9-->\text { high energy discharging }\end{array}$ & $3.15 \mathrm{E}-02$ & 1 \\
\hline $\begin{array}{c}\mathrm{CH} 4<79 \wedge \mathrm{C} 2 \mathrm{H} 6<7 \wedge \mathrm{C} 2 \mathrm{H} 4<26.1 \wedge \mathrm{C} 2 \mathrm{H} 2>20.9--> \\
\text { high energy discharging }\end{array}$ & 4.72E-02 & 0.67 \\
\hline $\begin{array}{c}79<\mathrm{CH} 4<256 \wedge 7<\mathrm{C} 2 \mathrm{H} 6<15 \wedge \mathrm{C} 2 \mathrm{H} 4>76.9 \wedge \\
\mathrm{C} 2 \mathrm{H} 2>20.9-->>\text { high energy discharging }\end{array}$ & $3.15 \mathrm{E}-02$ & 1 \\
\hline $\begin{array}{c}\mathrm{CH} 4>256 \wedge 15<\mathrm{C} 2 \mathrm{H} 6<116 \wedge \mathrm{C} 2 \mathrm{H} 4>76.9 \wedge \\
6.1<\mathrm{C} 2 \mathrm{H} 2<20.9-->\text { hyperthermia and superheating }\end{array}$ & 4.72E-02 & 1 \\
\hline $\begin{array}{c}\mathrm{CH} 4>256 \wedge \mathrm{C} 2 \mathrm{H} 6>116 \wedge \mathrm{C} 2 \mathrm{H} 4>76.9 \wedge \\
6.1<\mathrm{C} 2 \mathrm{H} 2<20.9-->\text { hyperthermia and superheating }\end{array}$ & $3.15 \mathrm{E}-02$ & 1 \\
\hline $\begin{array}{c}51<\mathrm{H} 2<89 \wedge 79<\mathrm{CH} 4<256 \wedge 7<\mathrm{C} 2 \mathrm{H} 6<15 \wedge \\
\mathrm{C} 2 \mathrm{H} 4<76.9 \wedge \mathrm{C} 2 \mathrm{H} 2<6.9-->\text { normal }\end{array}$ & 4.35E-02 & 1 \\
\hline $\begin{array}{c}51<\mathrm{H} 2<89 \wedge \mathrm{CH} 4>256 \wedge 15<\mathrm{C} 2 \mathrm{H} 6<116 \wedge \\
\mathrm{C} 2 \mathrm{H} 4>76.9 \wedge 6.1<\mathrm{C} 2 \mathrm{H} 2<20.9-->\text { hyperthermia } \\
\text { and superheating }\end{array}$ & $6.15 \mathrm{E}-02$ & 1 \\
\hline $\begin{array}{c}\mathrm{H} 2>318 \wedge 79<\mathrm{CH} 4<256 \wedge 15<\mathrm{C} 2 \mathrm{H} 6<116 \wedge \\
26.1<\mathrm{C} 2 \mathrm{H} 4<76.9 \wedge \mathrm{C} 2 \mathrm{H} 2>20.9-->\text { low energy } \\
\text { discharging }\end{array}$ & $3.25 \mathrm{E}-02$ & 0.87 \\
\hline $\begin{array}{c}89<\mathrm{H} 2<191 \wedge 79<\mathrm{CH} 4<256 \wedge \mathrm{C} 2 \mathrm{H} 6>116 \wedge \\
\mathrm{C} 2 \mathrm{H} 4>76.9-->\text { medium temperature and } \\
\text { superheating }\end{array}$ & $3.85 \mathrm{E}-02$ & 1 \\
\hline $\begin{array}{c}\mathrm{H} 2<20 \wedge \mathrm{CH} 4<79 \wedge 7<\mathrm{C} 2 \mathrm{H} 6<15 \wedge \mathrm{C} 2 \mathrm{H} 4<26.1 \wedge \\
\mathrm{C} 2 \mathrm{H} 2<1.1-->\text { normal }\end{array}$ & 4.54E-02 & 1 \\
\hline
\end{tabular}

All of the refined rules are placed in the knowledge base waiting for use. Once a new set of key gas values of a power transformer is provided, we can diagnose the working state of the power transformer by matching the rules in the knowledge base. Fig.2. shows an interface of fault diagnosis.

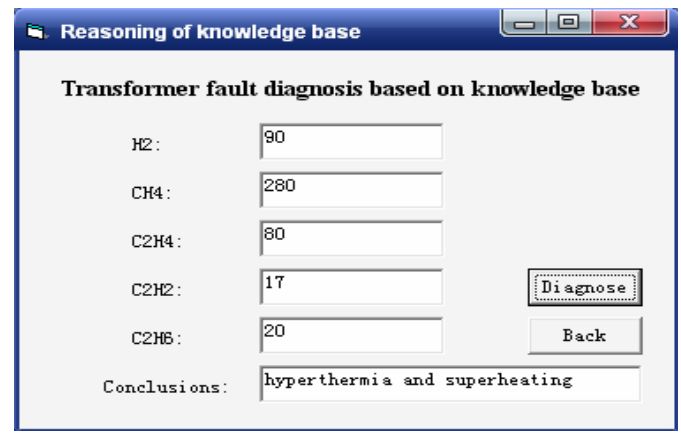

Figure 2 Fault diagnosis based on knowledge base

To validate the proposed approach, we used the IEC three-ratio method to diagnose the fault of power transformers with the same test data sets. The IEC threeratio method is widely accepted and used in on-site work, so the comparison basically can show the performance of the proposed method. The proposed approach using the refined rules in Table VI diagnosed 73 test data sets and the IEC three-ratio method also is used for comparision, the results shown in Table VII.

From Table VII, we can see that the proposed approach using association rule achieved higher accuracy in each fault type, especially for hyperthermia and superheating, the accuracy reached $100 \%$, although it's expected to be identified by more experiments.

TABLE VII. COMPARISON RESULTS

\begin{tabular}{|c|c|c|c|}
\hline \multirow{2}{*}{ Fault type } & \multirow{2}{*}{$\begin{array}{c}\text { Number of } \\
\text { Samples }\end{array}$} & \multicolumn{2}{|c|}{ Accuracy (\%) } \\
\cline { 3 - 4 } & 33 & 81.8 & 90.9 \\
\hline Normal & 10 & 80.0 & 100.0 \\
\hline $\begin{array}{c}\text { Hyperthermia and } \\
\text { superheating }\end{array}$ & 10 & 70.0 & 90.0 \\
\hline High energy discharging & 10 & 70.0 & 80.0 \\
\hline Low energy discharging & 10 & 80.0 & 80.0 \\
\hline $\begin{array}{c}\text { Medium temperature and } \\
\text { superheating }\end{array}$ & 73 & 78.1 & 89.0 \\
\hline Total & & & \\
\hline
\end{tabular}

\section{CONCLUSION AND FUTURE WORK}

Power transformer fault is a complicated problem and the development of related research is important. This poses a challenge, for which we proposed an approach based on ARM and the knowledge base. In the approach, the modified Apriori algorithm is used to generate rules, and then the rules are refined by a domain expert and stored in a knowledge base. When a new power transformer's data is provided, the rules in knowledge base will be searched and matched to diagnose the fault. The results of comparison with the IEC three-ratio method show that the use of association rules for fault diagnosis is feasible and generates better performance. Moreover, the application of the knowledge base is useful to incorporate empirical results of 
field experts or other resources.

Our future work includes developing some methods to assist domain expert to refine the rules process, and to find rule selection technologies to use the rules in the knowledge base appropriately and solve the rule contradictions. Future work will consider more related information and collect more fault records about power transformers; so that we can better improve the result of ARM.

\section{REFERENCES}

[1] IEEE, IEEE Guide for the Interpretation of Gases Generated in Oil Immersed Transformers, standard ed. Piscataway, NJ: IEEE Press, 1992.

[2] R. Rogers and B Barroclough, "CEGB Experience of the Analysis of Dissolved Gas in Transformer Oil for the Detection of Incipient Faults," Diagnostic Testing of High Voltage Power Apparatus in Service Conference, 1973, pp.128-130.

[3] H. Xiong, "Application of Fuzzy Rough Set Theory to Power Transformer Faults Diagnosis," Proceedings of the CSEE, Vol.28, No.7, pp.141-147, 2008 .

[4] X. X. Zheng and J. X. Wang, "Power transformer fault diagnosis based on variable precision rough set," Electric Utility Deregulation and Restructuring and Power Technologies, Third International Conference on 6-9 April, 2008, pp.1353-1358.

[5] F. Yang, "Fuzzy Theory and Probability Reasoning Applied for Identifying Power Transformer Fault," High Voltage Engineering, Vol.34, No.5, pp.34-36, 2008.

[6] Q. Su, C. Mi, L.L. Lai and P. Austin, "A fuzzy dissolved gas analysis method for the diagnosis of multiple incipient faults in a transformer," IEEE Transactions on Power Systems, Vol.15, No. 2, pp.593-598, May 2000.

[7] W. Niu, L. F. Xu and J.L. Wu, "Fault diagnosis and system development of power transformer based on support vector machine," Computer Science and Information Technology, 2nd IEEE International Conference on 8-11 Aug. 2009, pp.578-581.

[8] A. Salami and P. Pahlevani, "Neural network approach for fault diagnosis of transformers," Condition Monitoring and Diagnosis International Conference on 21-24 April 2008, pp.1346-1349.

[9] D.V.S.S. Siva and G.N.S. Sarma, Kalyani, "ANN approach for condition monitoring of power transformers using DGA," TENCON.IEEE Region 10 Conference Volume C, 21-24 Nov. 2004, pp. $444-447$.

[10] J. L. Guardado, J. L. Naredo and P. Moreno, "A comparative study of neural network efficiency in power transformers diagnosis using dissolved gas analysis," IEEE Transactions on Power Delivery, Vol.16, No.4, pp.643-647, October 2001.

[11] Y. Zhang, X. Ding and Y. Liu, "An artificial neural network approach to transformer fault diagnosis," IEEE Transactions on Power Delivery, Vol. 11, No. 4, pp.1836-1841, October 1996.

[12] J.G. Rolim,. P.C Maiola,. H.R. Baggenstoss and A.R.G.da Paulo, "Bayesian Networks Application to Power Transformer Diagnosis," Power Tech, 2007 .IEEE Lausanne 1-5 July 2007, pp.999-1004.

[13] Z.H. Peng and B. Song, "Application of Data Mining Technique Based on Grey Relational Analysis in Oil-Immersed Power Apparatus Fault Diagnosis," Power System Technology International Conference on 22-26 Oct. 2006, pp.1-4.

[14] M.H. Wang, "A novel extension method for transformer fault diagnosis," IEEE Transactions on Power Delivery, Vol. 18, No. 1, pp.164-169, Jan 2003.

[15] J.L. Sheng,. M.S. Zhou, Z.P. Guo and Z. Liu, "Fault diagnosis for transformer based on fuzzy entropy," Electrical Insulation and Dielectric Phenomena Annual Report - Conference on 14-17 Oct. 2007, pp.759-762.

[16] Z. Qian, W.S. Gao and Z. Yan, "Application of Case-based Reasoning Theory in Fault Diagnosis of Power Transformer," Properties and applications of Dielectric Materials 8th International Conference on June 2006, pp.242-245.
[17] F. Zhao and H.S. Su, "A decision tree approach for power transformer insulation fault diagnosis," Intelligent Control and Automation, 7th World Congress on 25-27 June 2008, pp.6882-6886.

[18] H. Xiong, T. Chang,. R.J. Liao, J. Li and C.X. Sun, "Fault Diagnosis of Power Transformer Using Kernel-Based Possibilistic Clustering," Power System Technology International Conference on 22-26 Oct. 2006, pp.1-5.

[19] M. Ahfaz Khan, "Expert System for Power Transformer Condition Monitoring and Diagnosis," IEEE International Conference on Power Electronics, Drives and Energy Systems International Conference on 12-15 Dec. 2006, pp.1-6.

[20] K.V. Satyanarayana, C.C. Reddy, T.P. Govindan, M. Mandlik and T.S. Ramu, "Application of Artificial Intelligence for the Assessment of the Status of Power Transformers," Electrical Insulation Conference Record of IEEE International Symposium on 9-12 June 2008, pp.104- 107

[21] Y.H. Gong and B. Zhang, "The Fault Diagnosis Model of Transformer Which Based on the Technology of Information Fusion," Hybrid Intelligent Systems, Fifth International Conference on12-14 Aug. 2009, Volume 1, pp.184-187.

[22] H.C. Shu, Z.J. Hu, S.Y. Sun and Q. Yang, "The fault diagnosis algorithm for transformer based on Extenics and rough set theory," Electric Utility Deregulation and Restructuring and Power Technologies, Third International Conference on 6-9 April 2008, pp.1269-1272.

[23] R. Naresh, V. Sharma and M. Vashisth, "An Integrated Neural Fuzzy Approach for Fault Diagnosis of Transformers," IEEE Transactions on Power Delivery, Vol. 23, No. 4, pp.2017-2024, Oct. 2008.

[24] H.Q. Sun, L.H. Sun, Y.C. Liang and Y.J. Guo, "The module fault diagnosis of power transformer based on GA-BP algorithm," Machine Learning and Cybernetics International Conference on 1821 Aug. 2005, Vol. 3, pp.1596-1598.

[25] A. Basel, A. F Mahafzah and Z. Mohammed, "A new sampling technique for association rule mining," Journal of Information Science, Vol. 35, No. 3, pp.358-376, 2009.

[26] Z. Yang, W. H. Tang, A. Shintemirov, and Q. H. Wu, "Association rule mining-based dissolved gas analysis for fault diagnosis of power transformers, " IEEE Transactions on Systems, Man, and Cybernetics, Part C: Applications and Reviews, Vol.39 No.6, pp.597610, November 2009.

[27] R. Agrawal and R. Srikant, "Fast algorithms for mining association rules," Proceedings of the 20th international conference of very large data bases, 1994, pp. 487-499.

[28] Z. Zheng, J. Lu and G. Zhang, "Rule sets based bilevel decision model and algorithm," Expert Systems with Applications, Vol. 36, No. 1, pp.18-26, 2009.

[29] T.F Zhang, L. Hua, L.S Liang, J. Lu and G. Zhang, “A Knowledgebased Efficiency Assessment System for Distribution Network using Data Envelopment Analysis," The Fourth International Conference on Research Challenges in Information Science (RCIS), Nice, France, May, 2010, pp.331-336

[30] J. Han and M. Kamber, Data Mining: Concepts and Techniques Morgan Kaufmann Morgan Kaufmann, 2 Edition, Nov.2005

[31] H.S. Nguyen and A. Skowron, "Quantization of Real Values Attributes, Rough set and Boolean Reasoning Approaches," .Proc. of the Second Joint Conference on Information Sciences on Oct. 1995, Wrightsville Beach, NC, pp. 34-37.

[32] http://www.lcb.uu.se/tools/rosetta/ 